\title{
Question word-order influences on covariate effects: predicting zero-sum beliefs
}

\author{
Michael Smithson ${ }^{1}$ (D) Yiyun Shou ${ }^{1} \cdot$ Alice \\ $\mathbf{Y u}^{1}$
}

Received: 3 July 2017/Accepted: 4 August 2017/Published online: 18 August 2017

(C) The Behaviormetric Society 2017

\begin{abstract}
Zero-sum-like statements have the form "The more of resource $\mathrm{X}$ for consumer A, the less of resource $\mathrm{Y}$ for consumer B" and these have four permutations, whereby $\{\mathrm{X}, \mathrm{A}\}$ and $\{\mathrm{B}, \mathrm{Y}\}$ exchange places, and/or "more" and "less" exchange places. Smithson and Shou (Front Psychol 7:984, 2016) found that these permutations strongly influence the endorsement of such statements. This paper reports two studies investigating personality traits as predictors of zero-sum endorsements, and whether their predictive performance is moderated by statement permutations. Social Dominance Orientation, Competitive World View, the Egocentricity subscale from the Levenson Self-Report Psychopathy Scale, and the Interpersonal subscale from the Hare Self-Report Psychopathy Scale are shown to be positively associated with zerosum endorsement, whereas none of the Big-5 personality factors are. Only the Egocentricity subscale's effect is moderated by statement permutations, so that for high scores on the subscale, zero-sum endorsement is boosted in such a way that the permutation effects disappear. The paper concludes with a discussion of the methodological implications of attitude statement permutation effects.
\end{abstract}

Keywords Questionnaire design · Word-order effect · Zero-sum · Measurement

Much has been written about the consequences of zero-sum thinking or fixed-pie beliefs have (e.g., Wright 2000). If a situation is perceived as zero-sum, those involved in negotiations or other joint activities are likely to act in competition, to

Communicated by: Kazuhisa Takemura.

Michael Smithson

Michael.Smithson@anu.edu.au

1 Research School of Psychology, The Australian National University, Bldg 39, Canberra, ACT 2601, Australia 
fail to consider others' viewpoints (Ross and Stillinger 1991), and positive outcomes for both parties are unlikely to result from negotiation (Gries 2005). A Forbes online columnist (Kalgaard 2006) called it the "worst disease" and laid its genesis at the feet of politicians and their advisors who "occupy a zero-sum world... in which one person's gain is another's loss." A key dividing-point between illiberal and liberal politics is whether wealth is zero-sum. Zero-sum perceptions also may contribute to inter-group prejudice (Bobo and Hutchings 1996) and public resistance to immigration (Esses et al. 2001).

However, until recently, little systematic investigation has been made into the causes and psychological triggers of zero-sum thinking or fixed-pie belief formation. The research that has been done on this topic has focused primarily on themes related to intergroup rivalry, competition, or threat. For example, Meegan (2010) suggests that zero-sum bias is linked to competitiveness. Smithson et al. (2015) studied how people subjectively evaluate degree of membership in national identities when presented with descriptions of migrants. They experimentally showed that people are more likely to allocate zero-sum membership assignments and lower combined membership in the country of origin and their adopted country to high-threat migrants than to low-threat migrants. In a four-nation study (USA, UK, India, and China), they also found that degree of membership in one's original nationality constrains the assignment of membership in a new nationality to a greater extent than vice versa.

Some researchers have pointed to intergroup status relations as triggering differential endorsement of zero-sum beliefs. Studies have reported that high-status group members tend to perceive status gains by low-status groups as entailing a loss of status for their own group. Norton and Sommers (2011) found that American White and Black respondents both believe that anti-Black discrimination has decreased, but White respondents also believe that there has been a corresponding increase in anti-White discrimination. Likewise, other researchers have found that men and women both believe anti-female bias has decreased, but men also believe that anti-male bias has increased (Bosson et al. 2012; Kehn and Ruthig 2013). None of these studies measured zero-sum beliefs directly, so it is unclear whether their findings involve genuinely zero-sum beliefs.

Wilkins et al. (2015) present a series of studies in which they manipulated perceptions of gender and racial bias and examined the effects on the endorsement of relevant zero-sum beliefs. They report the following main findings: (1) highstatus groups endorse zero-sum beliefs more than low-status groups; (2) high-status groups' endorsement of zero-sum beliefs increases when they perceive greater bias against their own group; and (3) endorsement of zero-sum beliefs correlates with efforts to improve high-status groups' outcomes and likewise with efforts to worsen low-status groups' outcomes.

Some findings in the literature suggest that personality, culture, and other individual differences traits also influence the tendency to perceive the world as a zero-sum place. Esses et al. (2001) report that individuals high in Social Dominance Orientation (SDO) are more likely to show zero-sum tendencies when expressing attitudes about immigration. Likewise, Wilkins et al. (Study 2b, 2015) report positive correlations between SDO and endorsement of both gender-specific and 
global zero-sum beliefs. In a more systematic investigation along these lines, Różycka-Tran et al. (2015) report a 37-nation scale-validation study of a "belief in zero-sum game" scale (BZSG, see Table 1). They find that individual-level measures of social trust, belief in balance of social exchanges, and self-esteem are negatively related to BZSG scores. The country-level covariates GDP and individualism are negatively related to BZSG scores, while societal cynicism is positively related to BZSG scores.

Before moving to further investigations along these lines, it is worthwhile to consider how best to measure zero-sum attitudes and/or beliefs. Różycka-Tran et al. (2015) measured zero-sum attitudes via the items listed in Table 1. In their multilevel CFA, they found the usual artefact regarding positive- and negative-direction items, namely that the positive items loaded on the first factor and the negative items on a second factor. This artefact is well known (e.g., Zimprich et al. 2005), despite the pervasive folk-wisdom throughout psychological research communities that a mix of positive and negative items is a hallmark of a good scale. RóżyckaTran et al. resolved this difficulty by removing the four negatively worded items from their scale. This is as far as many scale-construction exercises are taken.

However, there is an overlooked effect of zero-sum-type statements on respondents' apparent endorsement of them. Smithson and Shou (2016) study patterns of endorsements of zero-sum-like statements with the form "The more of resource $\mathrm{X}$ for consumer $\mathrm{A}$, the less of resource $\mathrm{Y}$ for consumer B". Several of the BZSG Scale items have this form. $\mathrm{X}$ and $\mathrm{Y}$ may be the same resource (such as time), but they can be different (e.g., "The more time it takes to produce a product, the lower the profit-margin for selling it"). Such statements have four permutations, whereby $\{\mathrm{X}, \mathrm{A}\}$ and $\{\mathrm{B}, \mathrm{Y}\}$ exchange places, and/or "more" and "less" exchange places. An example of these permutations are "Devoting more time to work takes time away from personal relationships"; "Devoting more time to personal relationships takes time away from work"; "Devoting less time to personal relationships leaves more time for work"; and "Devoting less time to work leaves more time for to personal relationships".

Table 1 BZSG Scale items

1. Successes of some people are usually failures of others

2. If someone gets richer, it means that somebody else gets poorer

3. Life is so devised that when somebody gains, others have to lose

4. In most situations, interests of different people are inconsistent

5. Life is like tennis game-a person wins only when others lose

6. When some people are getting poorer, it means that other people are getting richer

7. When someone does much for others, he or she loses

8. The wealth of a few is acquired at the expense of many

9. When a person does much for the good of others, he or she profits as well ${ }^{\mathrm{a}}$

10. Those who give much to others receive much from them ${ }^{\mathrm{a}}$

11. People who do much for their own good frequently benefit others as well ${ }^{\mathrm{a}}$

12. When the number of rich people increases in the country, the poorer people benefit as well ${ }^{\mathrm{a}}$

\footnotetext{
a These are negative-direction items
} 
A strict zero-sum interpretation would regard these four permutations as equivalent and, therefore, endorse them equally. Smithson and Shou (2016) find, however, that three strong framing effects routinely occur in people's endorsement levels, whereby endorsement of one permutation substantially differs from endorsement of another. For instance, in Smithson and Shou's (2016) Study 1, $87.1 \%$ of participants reported that they agreed more strongly with "Devoting more time to work takes time away from personal relationships" than with "Devoting more time to personal relationships takes time away from work". Moreover, these effects are reasonably consistent across three studies, with samples from adult populations in two Western and two non-Western cultures (USA, UK, India, and China). Smithson and Shou (2016) interpret the effects as arising from beliefs about asymmetric resource flows and power relations between rival consumers (see also Smithson et al. 2015).

Are these permutation effects important, and if so, what problems might they pose for determining the effects of covariates such as personality traits? Table 2 displays an example from Studies 1 and 2 in Smithson and Shou (2016). Study 1 asked participants to choose which of two statements they agreed with more, and in Studies 2 and 3 participants rated their degree of endorsement of each statement. The example in Table 2 is four pairwise comparisons between the four permutations of the statement "When the rich get richer, the poor get poorer". The percentagecomparisons from Study 1 are accompanied by the Study 2 odds-ratios of being rated in the upper part of the rating scale (odds-ratios are in parentheses). For example, in Study $183.2 \%$ of the participants chose "When the rich get richer, the poor get poorer" over "When the rich get poorer, the poor get richer" as the statement they agreed with more. "When the rich get poorer, the poor get richer" therefore was chosen by $17.8 \%$ of the participants, which is linked to the $83.2 \%$ figure by the box in the table. In Study 2, the first statement had 6.61 times higher odds than the second statement of being rated in the upper part of the rating scale.

Table 2 Percentages and odds-ratios for permutation comparisons

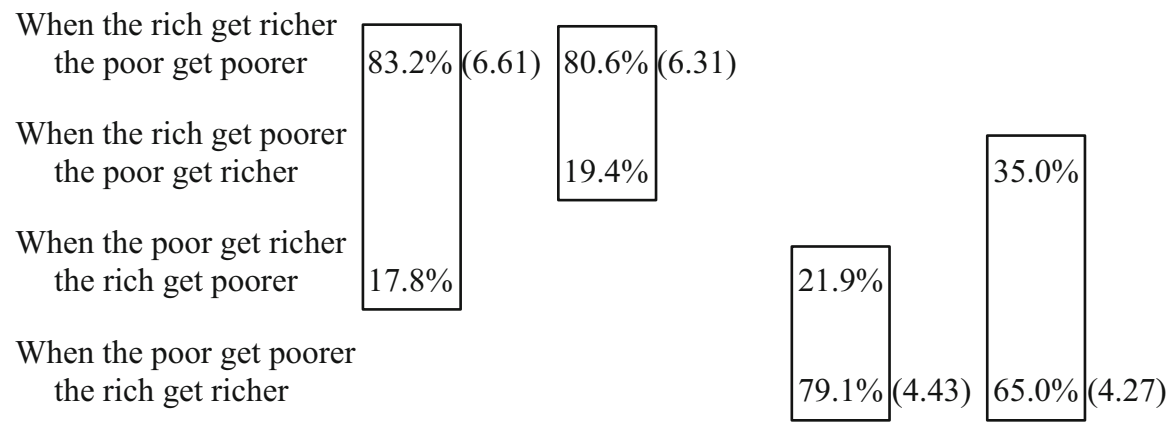


The magnitudes of these effects are substantial, and typical of the findings in Smithson and Shou (2016).

Given the sizeable impact of the permutations, it is plausible that they may moderate the relationship between any predictor of zero-sum beliefs and endorsement of zero-sum statements. If a trait, such as SDO, evokes strong zero-sum beliefs, then people scoring low on SDO will exhibit the permutation effects whereas high scorers will not. For example, low-scorers will endorse "When the rich get richer, the poor get poorer" more strongly than "When the rich get poorer, the poor get richer", whereas high-scorers will endorse both of these statements strongly and equally. These two patterns are illustrated schematically in Fig. 1, where the length of the grey bars represents the degree of statement endorsement. Therefore, the effect of SDO scores on statement endorsement level must be greater for the "When the rich get poorer, the poor get richer" permutation than for the "When the rich get richer, the poor get poorer" permutation.

Of course, it also is possible that the covariate influences operate independently of permutation effects on the strength of zero-sum belief. The point here is that the current literature does not distinguish between this pattern and the moderated covariate effect described above. In this paper, we extend the investigation of how individual differences variables predict zero-sum beliefs to several core psychological constructs, while assessing the permutation effects identified in the Smithson and Shou (2016) paper.

We already have reviewed literature suggesting a relationship between SDO and zero-sum beliefs. Given that intergroup competition triggers zero-sum beliefs, it is plausible that people who perceive the world as a competitive place will be more likely to endorse zero-sum beliefs than those who do not. Competitive World View (CWV) Scale measures a belief in a world that is characterised by a 'ruthless, amoral struggle for resources and power' (Perry et al. 2013). Hamilton and Rathbun (2013) propose that high scores on CWV are linked with a tendency to view international relations in zero-sum terms, especially regarding matters of national security.

Likewise, Social Values Orientation (SVO) measures the type of motivations or goals that people exhibit when distributing scarce resources between others and themselves. De Dreu and Boles (1998), examining the relationship between SVO and negotiation heuristics, classified zero-sum thinking as a type of competitive heuristic and suggested that a competitive SVO leads directly to this heuristic.

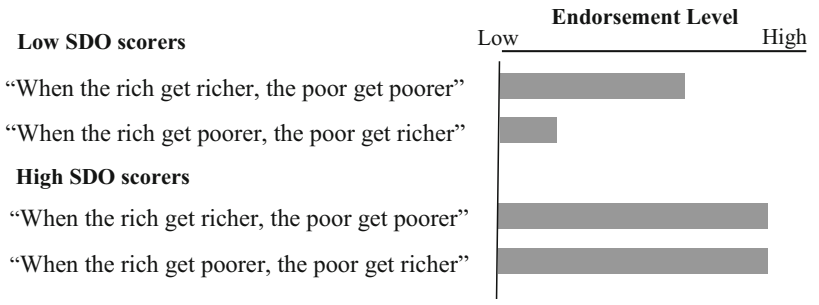

Fig. 1 Permutation moderating SDO effect 
We also investigate the relationship of zero-sum thinking to the "Big 5" personality model (McCrae and John 1992). Some interpretations of the factors (e.g., Wiggins 1996) have suggested that agreeableness may be related to tendencies toward cooperation and reciprocation, which in turn may be negatively related to zero-sum beliefs.

The studies reported in this paper are from the same samples as reported by Smithson and Shou (2016, their Studies 2 and 3), but here the focus is on the effects of individual difference variables on the endorsement of zero-sum statements, taking permutation effects into account. The main goals are to test hypotheses regarding these covariates, while ascertaining whether the covariate effects are moderated by permutation effects.

\section{Study 1}

The third author conducted a study prior to Study 1 (Yu 2014), as part of her Honours degree, with a sample of undergraduate students at The Australian National University (126 females and 65 males, $84 \%$ between 18 and 25 years old). She measured CWV, SDO, SVO, and the Big-5 factors as described in Study 1 below, and presented the zero-sum-like statements whose measurement properties were investigated by Smithson and Shou (2016). Using the sum of ratings on the eight zero-sum items as a dependent variable, in a linear regression model, she reported significant positive effects on zero-sum endorsement from CWV, SDO, and Conscientiousness. However, she found no significant main effects for SVO or the other Big-5 factors.

We repeated her study with adult samples from three countries (India, UK, USA), with a 50-50\% gender split. The main goal was to obtain adult samples from a variety of English-speaking populations with an equal gender split, greater agerange, and greater varieties of educational background and political orientations than is obtainable in a typical undergraduate sample. The hypotheses tested in this study are as follows:

H1.1: Endorsement of zero-sum statements will be positively related to SDO score

H1.2: Endorsement of zero-sum statements will be positively related to CWV score.

H1.3: Endorsement of zero-sum statements will be positively related to competitive SVO orientation.

H1.4: There will be no discernible relationship between endorsement of zero-sum statements and scores on any of the Big-5 personality factors (i.e., none of these factors will be associated with greater or lesser endorsement of zerosum statements, regardless of permutation). 


\subsection{Method}

\subsubsection{Participants}

There were 496 completed surveys by adult participants (at least 18 years old), from each of the three countries, with 50\% males in every sample. Participant data were excluded from the analyses if the participant spent less than $5 \mathrm{~min}$ on the survey, gave identical responses to all items, or did not answer "paying attention" questions correctly. The usable samples were as follows. The USA sample included 405 people, of whom $46.1 \%$ were males, and whose mean and standard deviation of age were 49.6 and 15.5, respectively. The UK sample included 426 people, of whom $49.3 \%$ were males, and whose mean and standard deviation of age were 46.6 and 18.2, respectively. The India sample included 452 people, of whom $51.8 \%$ were males, and whose mean and standard deviation of age were 37.5 and 17.2, respectively.

\subsubsection{Materials and design}

The study included two major parts, the zero-sum questions and a battery of items measuring the Big-5 personality factors (the BFI version: John et al. 1991), SVO, SDO (Ho et al. 2012), and CWV. The order of presentation of these two parts was counterbalanced, to control for any priming effect that either part might have on responses in the other.

The eight zero-sum-like statements in Study 1 initially were selected from a pilot study (Smithson and Shou 2016) in which participants were asked to choose between pairs of alternative permutations of various statements. Selection was based on how clear statements were to participants and an absence of unanimous agreement or disagreement with them. Studies 1 and 2 included four of the same zero-sum-like statements for purposes of replication. The zero-sum propositions were permuted in a between-subjects factor with four levels (see, Smithson and Shou 2016, for details). The overall design, therefore, was a 2 (survey-part order) $\times 4$ (permutations) factorial between-subjects experiment. Endorsement of the zero-sum propositions was measured by a 5-point Likert scale ranging from "strongly disagree" to "strongly agree". Examples of the permuted eight zero-sum propositions are displayed in Table 3.

\subsubsection{Procedure}

This study was conducted as an online experiment administered through a Qualtrics $^{\mathrm{TM}}$ panel, subsequent to approval by The Australian National University Human Research Ethics Committee. Participants were randomly assigned to one of the eight conditions. After an introductory screen asking for informed consent, the participants completed the individual differences measures and zero-sum proposition questions in the order determined by the condition to which they were assigned. Participants were then asked to provide their country of origin, English language 
Table 3 Study 1 Items and Examples

\begin{tabular}{|c|c|c|}
\hline Item & Label & Example \\
\hline $\mathrm{S} 1$ & Work-Personal & $\begin{array}{l}\text { Devoting more time to work takes time away from } \\
\text { personal relationships }\end{array}$ \\
\hline $\mathrm{S} 2$ & Distance-Time & $\begin{array}{l}\text { The longer the distance from } \mathrm{A} \text { to } \mathrm{B} \text {, the more time it takes to get } \\
\text { from A to B }\end{array}$ \\
\hline S3 & Eat-Weigh & The more I eat, the more I weigh \\
\hline S4 & Best Friend & $\begin{array}{l}\text { If my best friend increases the attention they pay to someone else, } \\
\text { they pay less attention to me }\end{array}$ \\
\hline S5 & Immigration & If the rate of immigration is increased there will be fewer jobs to go around \\
\hline S6 & Rich-Poor & When the rich get richer the poor get poorer \\
\hline S7 & Food-Clothes & Spending more money on clothes means there is less money to spend on food \\
\hline S8 & Cloudy-Sunny & The more hours of cloudy weather, the fewer hours of sunshine in a day \\
\hline
\end{tabular}

background, education level, age, and gender, after which they were thanked and debriefed via a closing information screen.

\subsubsection{Analysis}

The response variables are categorical ordinal variables and, therefore, have been analysed via mixed ordinal regression, the cumulative logit approach, using the VGAM package in R (Yee 2010) and the NLMIXED procedure in SAS. Owing to the large sample sizes, a significance criterion of 0.01 rather than the conventional 0.05 was adopted for model comparison purposes via likelihood-ratio tests.

\subsection{Results}

All models included main effects for the country from which the sample came, and main effects for zero-sum items. We list a summary of the main results below, and then elaborate those that require it.

1. There is no significant order effect, in presenting the zero-sum items first versus after the individual differences items $\left(\chi^{2}(3)<3\right.$, ns), so order effect was not included in subsequent models.

2. None of the covariate effects are significantly moderated by item permutation. Separate models for each of the seven covariates and each of the zero-sum items were run to test for moderation of the covariate effect by item permutation (49 models in total). None of them yielded moderator effects that were significant at the 0.01 level (just 3 of them had small effects with $0.01<p<0.05$, approximately a chance-level occurrence), and so permutation effects were omitted from subsequent models.

3. None of the effects are moderated by country (evidence for this is presented in the subsections dealing with each covariate). 
4. CWV has a positive main effect, which is moderated by item. For the Immigration and Best Friend items, it is substantially amplified. For the FoodClothes item, it is reversed, and for the Sunny-Cloudy item it is non-significant.

5. SDO has a positive main effect, which is moderated by item. The effect is amplified for the Distance and Immigration items, but almost eliminated for the Food-Clothes item.

6. SVO does not have a significant effect. For a main-effects model for SVO the fit improvement is not significant $\left(\chi^{2}(1)=6.11, p=0.013\right)$. We conclude that the SVO effect is marginal and not worth including, although it is in the expected direction.

7. There are no significant main effects for the Big-5 covariates. There are small moderator effects involving agreeableness, extraversion, and openness for just two items, but they are of no relevance to this paper and are not considered further.

The covariates were centred and entered one at a time into the model, first testing for a main effect, and then testing for moderator effects from country and from items. The final model (with centred covariates CWV and SDO) is presented in Eq. (1):

$$
\begin{aligned}
\operatorname{logit}\left(P\left(Y_{i q}>k\right)\right)= & \alpha_{k}+\beta_{00}+\sum_{j=1}^{2} \beta_{0 j} C_{j}+\sum_{m=2}^{8} \beta_{m} S_{m}+\delta_{0} \mathrm{CWV}_{i} \\
& +\gamma_{0} \mathrm{SDO}_{i}+\sum_{m=2}^{8} \delta_{m} S_{m} \mathrm{CWV}_{i}+\sum_{p=2}^{8} \gamma_{p} S_{p} \mathrm{SDO}_{i}+u_{i}+\varepsilon_{i q}
\end{aligned}
$$

The $\alpha_{k}$ are the threshold parameters in a cumulative logit model, and $\beta_{00}$ is the intercept for the combination of item S1 and country USA. The $C_{j}$ are dummyvariables taking values $\{0,1\}$ for the countries India and UK. The $S_{m}$ are effectscoded dummy-variables taking values $\{-1,0,1\}$ for items $\mathrm{S} 1-\mathrm{S} 7 . \mathrm{CWV}_{i}$ and $\mathrm{SDO}_{i}$ denote the CWV and SDO scale scores for the $i$ th subject, respectively.

\subsubsection{Competitive world view}

CWV has a main effect with no moderator effects from country $\left(\chi^{2}(2)<2\right.$, ns). Table 4 displays the relevant coefficients for CWV, along with their odds-ratio counterparts. The "+item" column adds the CWV coefficient to the item coefficient. For instance, the +item effect for $\mathrm{S} 1$ is $0.129-0.025=0.104$, and the resulting odds-ratio is $\exp (0.104)=1.11$, i.e., participants scoring higher on CWV endorsed S1 more strongly than low scorers. The positive main effect of CWV is nearly unchanged for items S1, S2, S3, and S7. Thus, at one standard deviation above the mean of CWV, the cumulative odds of endorsing an item increase by a factor of approximately 1.14. For items S4 and S6 (Best Friend and Rich-Poor), this effect is substantially amplified. For item S5, it is reversed, and for item S8 it is eliminated. Thus, for only two items is the CWV effect inapplicable: Items S5 and S8 (Immigration and Cloudy-Sunny). 
Table $4 \mathrm{CWV}$ and item moderator effects

\begin{tabular}{lrrrl}
\hline Item & \multicolumn{1}{c}{ Coeff. } & + Item & \multicolumn{1}{l}{$p$} & Odds-ratio \\
\hline CWV & 0.129 & & $<0.001$ & 1.137 \\
S1 Work-Personal & -0.025 & 0.104 & 0.651 & 1.110 \\
S2 Distance-Time & -0.030 & 0.098 & 0.573 & 1.103 \\
S3 Eat-Weigh & 0.019 & 0.148 & 0.713 & 1.160 \\
S4 Best Friend & 0.182 & 0.311 & 0.001 & 1.364 \\
S5 Immigration & -0.266 & -0.137 & $<0.001$ & 0.872 \\
S6 Rich-Poor & 0.267 & 0.396 & $<0.001$ & 1.486 \\
S7 Food-Clothes & 0.054 & 0.182 & 0.320 & 1.200 \\
S8 Cloudy-Sunny & -0.201 & -0.073 & $<0.001$ & 0.930
\end{tabular}

\subsubsection{Social Dominance Orientation}

SDO has a main effect with no moderator effects from country $\left(\chi^{2}(2)=3.44\right.$, $p=0.179$ ). Table 5 displays the relevant coefficients for SDO, along with their odds-ratio counterparts. For most of the items, there is little moderation of the SDO effect. This effect is stronger than that for CWV, so at one standard deviation above the mean of CWV, the cumulative odds of endorsing an item increase by a factor of approximately 1.38. The exceptions are items S1, S4, and S5. The effect is amplified for item S1 and marginally for item S4 (Work-Personal and Best Friend), and attenuated for item S5 (Immigration).

\subsection{Study 1: discussion}

The fact that the item permutations did not discernibly moderate covariate effects is a striking finding, especially considering the large magnitudes of the permutation effects on zero-sum statement endorsement levels. It suggests that individualdifference traits such as SDO or CWV and the permutation-relevant cognitive heuristics identified by Smithson and Shou (2016) may influence the endorsement of zero-sum-like statements independently of one another.

Table 5 SDO and item moderator effects

\begin{tabular}{lrrrl}
\hline Item & Coeff. & +Item & \multicolumn{1}{l}{$p$} & Odds-ratio \\
\hline SDO & 0.322 & & $<0.001$ & 1.380 \\
S1 Work-Personal & 0.138 & 0.460 & 0.005 & 1.584 \\
S2 Distance-Time & -0.017 & 0.305 & 0.758 & 1.357 \\
S3 Eat-Weigh & -0.041 & 0.281 & 0.435 & 1.324 \\
S4 Best Friend & 0.128 & 0.450 & 0.016 & 1.568 \\
S5 Immigration & -0.243 & 0.079 & $<0.001$ & 1.082 \\
S6 Rich-Poor & 0.024 & 0.346 & 0.640 & 1.413 \\
S7 Food-Clothes & 0.048 & 0.370 & 0.369 & 1.448 \\
S8 Cloudy-Sunny & -0.038 & 0.284 & 0.477 & 1.329 \\
\hline
\end{tabular}


SDO and CWV produced effects as hypothesized, but SVO's effect was not apparent. One possible explanation for the latter result is that the SVO measure is not highly reliable or stable (Bekkers 2004). None of the Big-5 measures yielded discernible effects, so perhaps core personality factors in normalfunctioning populations do not influence zero-sum beliefs. However, the SDO and CWV results indicate that a self-centred view of the world as a contest with the self as one of the "winners" may be a key influence in the formation of zero-sum beliefs. These results, therefore, also suggest that traits such as narcissism or ego-centrism of the psychopathological kind might be positively associated with endorsement of zero-sum beliefs. This is the focus of Study 2, along with the question of whether statement permutations moderate the impact of ego-centrism.

\section{Study 2}

In this study, we investigate the potential connection between subscales of psychopathy inventories (see below) and zero-sum belief endorsement. The major hypothesis is:

H2.1: Higher scores on both psychopathy inventories will predict greater zerosum statement endorsement

As in Study 1, we counterbalanced the order of presentation of the psychopathy items and zero-sum statements, to control for potential priming effects.

We advance no hypotheses regarding the potential moderation by items or item permutations of the effect that psychopathy scores may have on zero-sum belief endorsement because, as in Study 1, there are no theoretical bases for such hypotheses. Instead, this is an exploratory part of the study. That said, there is a pattern of results that is of particular interest here, namely whether high scores on psychopathy predict not only greater endorsement of zero-sum beliefs but also more equal endorsement levels across the four permutations (as illustrated in Fig. 1). Recall that a zero-sum believer should regard all four permutations as equally valid, so if psychopathic tendencies include a zero-sum worldview, then the permutation moderator effects should yield the pattern described here.

Study 2 also was designed to test the generality of the results from Study 1 in two respects. First, the rating scale was expanded from 5 to 7 bins, to test whether any of the patterns identified in the previous studies might be artefacts of the scale response format. To do this, four of the Study 1 statements were included in Study 2 and adult samples were obtained again from the USA, UK, and India. Second, cross-cultural generality was further tested by translating the items into Chinese and obtaining a sample of adult Chinese responses. The goal here was to explore whether the same patterns would emerge when the zero-sum statements are expressed in a language unrelated to English. 


\subsection{Method}

\subsubsection{Participants}

As in the preceding study, participant data were excluded from the analyses if the participant spent less than 5 min on the survey, "flat-lined" responses, or did not answer "paying attention" questions correctly. However, in this study, we recruited participants until we had attained very close to an even gender split and 500 participants in each country fulfilling these criteria. The USA sample included 498 people, whose mean and standard deviation of age were 45.8 and 15.6, respectively. The UK sample included 494 people, whose mean and standard deviation of age were 51.6 and 14.4, respectively. The India sample included 503 people, whose mean and standard deviation of age were 36.4 and 14.2, respectively. The China sample included 496 people, whose mean and standard deviation of age were 32.3 and 7.8 , respectively.

\subsubsection{Materials, design, procedure, and analysis}

As in Study 1, this study included two major parts, the zero-sum questions and a set of individual differences covariates, the findings for which are reported in Smithson and Shou (2016). The covariates consisted of subscales of two psychopathy inventories (see below). The order of presentation of the zero-sum items and psychopathy inventories was counterbalanced in each of the four samples.

Table 6 displays the zero-sum propositions used in this study. Two of them (S1 and $\mathrm{S} 4$ ) are replicates of statements from the pilot study reported by Smithson and

Table 6 Study 2 items

\begin{tabular}{|c|c|c|}
\hline Item & Label & Example $(\mathrm{Ab})$ \\
\hline So & Al-Bayati & $\begin{array}{l}\text { Consider Hama Al-Bayati, who immigrated to the USA } \\
\text { (UK, India) } 5 \text { years ago from Iraq. The more "Iraqi" he is } \\
\text { (A), the less "American" ("British", "Indian") he will be (b) }\end{array}$ \\
\hline S1 & Investment & $\begin{array}{l}\text { Investing more money in one venture (A) means there is less for } \\
\text { the others (b) }\end{array}$ \\
\hline $\mathrm{S} 2$ & Work-Personal & $\begin{array}{l}\text { Devoting more time to work (A) takes time away from personal } \\
\text { relationships (b) }\end{array}$ \\
\hline $\mathrm{S} 3$ & Immigration & $\begin{array}{l}\text { If the rate of immigration is increased (A) there will be fewer jobs to } \\
\text { go around (b) }\end{array}$ \\
\hline S4 & Friends-Family & Spending more time with friends (A) takes time away from family (b) \\
\hline S5 & Best Friend & $\begin{array}{l}\text { If my best friend increases the attention they pay to someone else (A), } \\
\text { they pay less attention to me (b) }\end{array}$ \\
\hline S6 & Rich-Poor & When the rich get richer (A) the poor get poorer (b) \\
\hline S7 & Al-Husseni & $\begin{array}{l}\text { Consider Ali Al-Husseni, who immigrated to Germany } 5 \text { years ago } \\
\text { from Iraq. The more "Iraqi" (A) he is (A), the less "German" } \\
\text { he will be (b) }\end{array}$ \\
\hline
\end{tabular}


Shou (2016) that were not retested in Study 1. Four others (S2, S3, S5, and S6) are replicates of items from Study 1. The remaining two (S0 and S7) are new items, designed to test hypotheses regarding marginalizing ethnocentrism (in Smithson et al. 2015). Endorsement of the zero-sum propositions was measured by a 7-point Likert scale ranging from "strongly disagree" to "strongly agree".

Table 6 also introduces notation employed by Smithson and Shou (2016), in which resource consumers are identified by letter (A and B). An upper-case letter denotes an increase in a resource for the consumer, whereas a lower-case letter denotes a decrease in the resource. For instance, "Ab" denotes the more of a resource for consumer $\mathrm{A}$, the less of it for consumer $\mathrm{B}$, whereas " $\mathrm{aB}$ " denotes the less of a resource for consumer A, the more of it for consumer B. Based on the findings reported by Smithson and Shou (2016), the Ab permutations in Table A are generally more strongly endorsed than the other combinations. Regarding S2, for instance, "Devoting more time to work takes time away from personal relationships" (Ab) is endorsed more strongly than "Devoting more time to personal relationships takes time away from work" (Ba).

As in Study 1, response variables are categorical ordinal variables and, therefore, have been analysed via mixed ordinal regression using the VGAM package in $\mathrm{R}$ (Yee 2010) and the NLMIXED procedure in SAS. Again, a significance criterion of 0.01 rather than the conventional 0.05 was adopted for model comparison purposes via likelihood-ratio tests.

The Levenson Self-Report Psychopathy Scale (LSRP; Levenson et al. 1995; revised version, Brinkley et al. 2008) and the Hare Self-Report Psychopathy Scale (SRP-II; Hare et al. 1989; revised version, Lester et al. 2012) were used to measure the core of psychopathic traits.

The LSRP, consisting of 26 items, is an efficient measure of psychopathy and has shown promising psychometric properties in a range of university, forensic, correctional, and general community samples (see Sellbom et al. 2017, for a review). A three-factor model that captures Egocentricity, Callous, and Antisocial factors was proposed by Brinkley et al. (2008), and has been validated in various samples, including Chinese samples (Sellbom et al. 2017; Shou et al. 2016). The Egocentricity scale was selected in the present study for their conceptual relevance to zero-sum thinking. The Egocentricity scale (11 items) reflects the maladaptive interpersonal functioning, and captures characteristics including selfishness, narcissism, dominance, and interpersonal manipulation. In our samples, Cronbach's $\alpha=0.86$.

The SRP-II was developed based on Hare's Psychopathy Checklist List (PCL; Hare 1991), and contains two facets of psychopathy. The first captures the core of psychopathy featured by maladaptive interpersonal and affective trait, while the second facet measures the antisocial tendency. Recent studies suggest that a fourfactor model can be more adequate than the original two facet structure (Lester et al. 2012; Sellbom et al. 2017). The four factors include (1) Interpersonal, (2) Coldheatedness, (3) Fearlessness; and (4) Disinhibition. The first two correspond to the primary facet, while the other two correspond to the second facet. For the reason mentioned above, we chose the Interpersonal subscale in the present study. The 
Interpersonal subscale (16 items) assesses characteristics of dominance, manipulativeness, and arrogance. In our samples, for this scale Cronbach's $\alpha=0.82$.

\subsection{Results}

As before, all models included main effects for country and main effects for zerosum items. The covariates were centred and entered one at a time into this basic model, first testing for a main effect, and then testing for moderator effects from country and from items and/or item permutations. The final model may be written as:

$$
\begin{aligned}
\operatorname{logit}\left(P\left(Y_{i q}>k\right)\right)= & \alpha_{k}+\beta_{00}+\sum_{j=1}^{3} \beta_{0 j} C_{j}+\sum_{m=1}^{7} \beta_{m} S_{m}+\delta_{0} \mathrm{SRP}_{i} \\
& +\gamma_{0} \operatorname{LSRP}_{i}+\sum_{p=1}^{7} \gamma_{p} S_{p} \operatorname{LSRP}_{i} \\
& +\phi_{0} \operatorname{Order}_{i}+\sum_{p=1}^{7} \phi_{p} S_{p} \operatorname{Order}_{i}+u_{i}+\varepsilon_{i q} .
\end{aligned}
$$

The $\alpha_{k}$ are the threshold parameters in a cumulative logit model, and $\beta_{00}$ is the intercept for the combination of item S0 and country USA. The $C_{j}$ are dummyvariables taking values $\{0,1\}$ for the countries India, UK, and China. The $S_{m}$ are effects-coded dummy-variables taking values $\{-1,0,1\}$ for items S1-S7. SRP $2_{i}$ and $\mathrm{LSRP}_{i}$ denote the SRP-II and LSRP scale scores for the $i$ th subject, respectively. Order $_{i}$ is a dummy variable equalling 1 if the psychopathy items were presented first and -1 if it did not. This model's fit does not significantly differ from a model with SRP2*item interaction terms $\left(\chi^{2}(7)=5.01, p=0.660\right)$. Thus, while the effect of LSRP Egocentricity was moderated by items, the effect of SRP Interpersonal was not.

As before, we present a summary list of the main results and then elaborate those as required.

1. The LSRP Egocentricity scale positively predicts endorsement of zero-sum beliefs for all items. Items moderate the effect of LSRP Egocentricity.

2. There are item-permutation moderator effects involving LSRP Egocentricity for four of the eight items, and these were estimated in separate item-by-item analyses subsequent to the main model reported above.

3. The SRP Interpersonal scale positively predicts endorsement of zero-sum beliefs. Items do not moderate the effect of SRP Interpersonal; nor do permutations of items.

4. When the psychopathy items are presented first, they decrease endorsement in six items, the exceptions being the Immigration and Rich-Poor items. This effect is amplified for the Investment item. 


\subsubsection{LSRP Egocentricity and SRP Interpersonal effects}

The main effects for SRP Interpersonal and LSRP Egocentricity both are positive, i.e., higher scores on either scale predict stronger endorsement of zero-sum statements. One standard deviation increase in the SRP Interpersonal scale predicts 1.141 times greater cumulative odds of zero-sum endorsement $\left(\hat{\delta}_{0}=0.132\right.$, $p<0.001, \exp (0.132)=1.141)$.

The average effect for the LSRP Egocentricity scale is greater $(\exp (0.236)=1.266)$, and this effect is moderated by item. Table 7 displays the item moderator effects, with significant effects for S2 (Work-Personal), S5 (Best Friend), and S6 (Rich-Poor), and a marginal effect for S4 (Friends-Family). The effect is eliminated for S2 and amplified for S5 and S6.

Turning now to the item-permutation moderator effects highlighted in the second finding on the list above, four of the items' permutation-moderator models showed significant moderators effects (i.e., $p<0.01$ in the likelihood-ratio test comparing a model with and without the moderator terms). Table 8 presents these effects by displaying the odds-ratios for each of the relevant items as a function of their permutation form. There is a clear tendency for the LSRP Egocentricity scores to have little or no influence on endorsement of the Ab permutation (their odds-ratios are close to 1 except for Work-Personal, where the odds actually decrease slightly), whereas endorsements of both the $\mathrm{Ba}$ and $\mathrm{bA}$ permutations show substantial influence by LSRP Egocentricity, and the aB permutation shows influence for three of the four items. All of these effects, in line with the major findings, indicate that higher LSRP Egocentricity scores increase endorsement of these permuted items.

\subsubsection{Priming effects}

The main effect for priming reduces the level of endorsement of zero-sum statements. The cumulative odds of endorsing an item decrease by a factor of 0.900 when the psychopathy scales' items are presented prior to the zero-sum statements (this is derived by exponentiating the mean of the $\phi$ coefficients in the model described above). As mentioned earlier, this effect is moderated by items. Table 9

Table 7 Item moderator effects for the LSRP Egocentricity scale effect

\begin{tabular}{lrrrrl}
\hline Item & \multicolumn{1}{l}{ Coeff. } & \multicolumn{1}{l}{ SE } & \multicolumn{1}{l}{ + Item } & Odds-ratio \\
\hline LSRP & 0.236 & 0.039 & $<0.001$ & & 1.2657 \\
S0 & -0.010 & 0.037 & 0.394 & 0.2254 & 1.2528 \\
S1 & -0.024 & 0.037 & 0.519 & 0.2116 & 1.2356 \\
S2 & -0.237 & 0.039 & $<0.001$ & -0.0015 & 0.9985 \\
S3 & 0.048 & 0.040 & 0.228 & 0.2832 & 1.3274 \\
S4 & -0.089 & 0.039 & 0.021 & 0.1464 & 1.1576 \\
S5 & 0.168 & 0.037 & $<0.001$ & 0.4036 & 1.4972 \\
S6 & 0.151 & 0.041 & $<0.001$ & 0.3864 & 1.4717 \\
S7 & -0.006 & 0.037 & 0.875 & 0.2298 & 1.2583 \\
\hline
\end{tabular}


Table 8 Item-permutation moderator effects odds-ratios for LSRP Egocentricity

a The heuristics are explained in the Study 2 Discussion section

Table 9 Item moderator effects for the priming effect

\begin{tabular}{llllll}
\hline Item & $\mathrm{Ab}$ & $\mathrm{Ba}$ & $\mathrm{aB}$ & $\mathrm{bA}$ & Heuristic $^{\mathrm{a}}$ \\
\hline S1 Work-Personal & 0.813 & 1.481 & 1.082 & 1.602 & $\mathrm{ACP}+$ ARF \\
S3 Immigration & 0.956 & 1.495 & 1.326 & 1.637 & $\mathrm{ACP}+\mathrm{ARF}$ \\
S4 Friend-Family & 0.957 & 1.336 & 1.201 & 1.436 & $\mathrm{ACP}+\mathrm{ARF}$ \\
S7 Al-Husseni & 1.046 & 1.523 & 1.496 & 1.454 & AGP \\
\hline
\end{tabular}

\begin{tabular}{lrlrrl}
\hline Item & Coeff. & \multicolumn{1}{l}{ SE } & \multicolumn{1}{l}{ +Item } & Odds-ratio \\
\hline S0 & -0.119 & 0.026 & $<0.001$ & & 0.888 \\
S0 & -0.112 & 0.037 & 0.001 & -0.231 & 0.794 \\
S1 & -0.096 & 0.037 & 0.009 & -0.215 & 0.807 \\
S2 & -0.009 & 0.038 & 0.817 & -0.128 & 0.880 \\
S3 & 0.089 & 0.038 & 0.021 & -0.030 & 0.970 \\
S4 & -0.061 & 0.037 & 0.104 & -0.180 & 0.836 \\
S5 & 0.043 & 0.036 & 0.234 & -0.076 & 0.927 \\
S6 & 0.179 & 0.040 & $<0.001$ & 0.060 & 1.062 \\
S7 & -0.033 & 0.036 & 0.359 & -0.152 & 0.859 \\
\hline & & & & &
\end{tabular}

presents the moderator effects and the resulting odds-ratios. The significant moderator effects are for items S0 (al-Bayati), S1 (Investment), and S6 (Rich-Poor) with a marginal effect for S3 (Immigration). The priming effect is eliminated for S6 and S3, whereas it is amplified for S0 and S1.

\subsection{Study 2 discussion}

As described in the preceding section, both the LSRP Egocentricity and SRP Interpersonal subscales are positively associated with endorsement of zero-sum beliefs, with the LSRP Egocentricity effect being both larger than that for SRP Interpersonal and moderated by items. The correlation between these two subscales in our samples was 0.69 , so they share a substantial portion of variance, but the LSRP Egocentricity scale emphasizes selfishness and narcissism in addition to the dominance and manipulation traits captured by both it and the SRP Interpersonal scale. Our findings suggest that selfishness and narcissism contribute to zero-sum belief over and above tendencies to dominate and manipulate other people.

The priming effect of the psychopathy scales, although not large, is detectable. Participants endorsed zero-sum belief statements less when they completed the psychopathy items beforehand rather than afterward, but the effect size is small enough that this finding holds more methodological than practical interest. It also is noteworthy that neither the SDO nor CWV scales produced priming effects. Both of those scales ask respondents to make attributions about others and social relations, whereas the psychopathy items reflect respondents' views about their own values and morals. It is possible that priming effects on zero-sum belief endorsements are limited to stimuli invoking thoughts about one's own selfishness or egocentricity. 
We now turn to the permutation moderation of the LSRP Egocentricity effect. Three interpretive issues require attention here: the nature of the permutation moderator effects, why some items yield moderator effects but not others, and why the LSRP Egocentricity effect is moderated but not SRP Interpersonal.

Interpreting the moderator effects requires understanding the original permutation effects. As shown in the right-most column of Table 8, three of the permutation moderator effects occur for items whose "heuristic" is labelled ACP + ARF and one item whose heuristic is labelled AGP. These were the two major permutationdriven endorsement patterns identified in Smithson and Shou (2016). ACP is "asymmetric consumer potency", in which one resource consumer is regarded as more potent or powerful than the other (e.g., a belief that spending more time at work decreases time for personal relationships but the converse is not true). ARF is "asymmetric resource flow", where a consumer increasing their share of the resource decreases other consumers' shares but decreasing their share does not necessarily increase others' shares (e.g., a belief that paying more attention to person $\mathrm{X}$ results in less attention to person $\mathrm{Y}$, but paying less attention to $\mathrm{X}$ does not increase attention to Y). Finally, AGP is "asymmetric gains potency", where gains in a resource have greater potency for one consumer than for another (e.g., a belief that the rich always get richer whereas the poor never do). The permutation moderator effects occur in three of the four ACP + ARF items in this study, but only for one of three AGP items.

The ACP + ARF endorsement pattern is that the Ab permutation is endorsed more strongly than $\mathrm{Ba}$ and $\mathrm{aB}$, while $\mathrm{aB}$ is more strongly endorsed than $\mathrm{bA}$. As suggested at the beginning of Study 2, if the effect of LSRP Egocentricity is to increase zero-sum belief then it should be eliminating these differences, so it should have its strongest effect on the $\mathrm{aB}$ permutation, followed by effects on $\mathrm{Ba}$ and $\mathrm{aB}$, with the weakest or no effect on Ab. For the three ACP + ARF items in Table 8, this is the pattern. Scoring high enough on LSRP Egocentricity suffices for someone to endorse all four permutations equally, which is equivalent to a full zero-sum belief. The AGP endorsement pattern has both $\mathrm{Ab}$ and $\mathrm{bA}$ endorsed more strongly than $\mathrm{Ba}$ and $\mathrm{aB}$. If the effect of LSRP Egocentricity is to increase zero-sum belief then its strongest effects should be on $\mathrm{Ba}$ and $\mathrm{aB}$, with weaker or no effects on $\mathrm{Ab}$ and $\mathrm{bA}$. Three permutations fit this pattern, but the bA permutation does not.

It appears that ARF + ACP items are more susceptible to permutation moderator effects than AGP items, although the evidence for this is far from conclusive. It also is puzzling that the Al-Husseni item (S7) yielded a permutation moderator effect whereas the Al-Bayati (S0) item did not. The difference between these two items is that the Al-Bayati target is an immigrant to the participant's own country, whereas the Al-Husseni target is an immigrant to Germany, which suggests that the AGP heuristic may be more stable when the destination country is not one's own.

Overall, then, the evidence suggests that ego-centrism as measured by the LSRP Egocentricity subscale is positively associated with a tendency to view the world in zero-sum terms. It is somewhat puzzling that the same kind of permutation moderator effect was not found for the SRP Interpersonal subscale. As mentioned earlier, the LSRP Egocentricity subscale emphasizes selfishness and narcissism in addition to dominance and manipulation, so perhaps these traits not only boost zero- 
sum belief but do so in a way that overrides the heuristics that cause people to differentially endorse zero-sum statements when their elements are permuted.

\section{General discussion}

As this is primarily a methodological paper presenting an empirical test-case, this final discussion section focuses on the issues raised by the findings in relation to Smithson and Shou's (2016) item-permutation effects. The central question being addressed here is whether item permutations moderate covariate or predictor effects. We have mixed findings, albeit with a majority of the covariates investigated herein showing little or no moderation by permutations of items, despite the large permutation main effects reported in Smithson and Shou (2016). The absence of these moderator effects from the impacts of SDO, CWV, and SRP Interpersonal on zero-sum belief endorsements is something of a relief, given that such effects introduce complications into the assessment of predictors of zero-sum belief. However, the moderation of the LSRP Egocentricity effects by permutations was sufficient in four items to eliminate this effect on item endorsement, so we have demonstrated that these moderator effects are worthy of attention.

Moreover, as Smithson and Shou (2016) observe in their concluding paragraph, permutation order effects may occur in many kinds of items. They may lurk in attitude measures containing multi-clause statements in which different orderings of the clauses alter the statement's meaning and/or implications. Commonplace examples of such items are conditional or modus-ponens statements (if $\mathrm{X}$ then $\mathrm{Y}$ ), causal statements (X causes $\mathrm{Y}$ ), and statements with temporal ordering ( $\mathrm{X}$ is followed by $\mathrm{Y}$ ). When permutation moderator effects occur, what are the consequences for experimental methods and measurement, and how may they be interpreted or integrated into theoretical developments?

The methodological consequences are fairly straightforward. First, experiments and scale-development studies must be designed to detect permutation effects. Second, both main and moderator permutation effects should be routinely tested in models fitted to the data. In scale construction, researchers may test for so-called measurement invariance under item permutations, or alternatively may argue for choosing a particular permutation of an item as most suitable for their scale.

Integrating permutation effects with psychological theory will, of course, require the development of theories about these effects. Smithson and Shou's (2016) approach is to conjecture and test lay interpretive heuristics that may lead to the permutation effects they have identified, but they do not provide an account that would enable researchers to predict which heuristic might apply to a particular zero-sum belief statement. The elaboration and testing of a theory of permutation effects therefore is an open topic for research in the science of questionnaire construction.

Acknowledgements Funding was provided by Australian Research Council (Grant No. DP120101095). 


\section{References}

Bekkers R (2004) Stability, reliability and validity of social value orientation. doi:10.2139/ssrn.2274560

Bobo L, Hutchings VL (1996) Perceptions of racial group competition: extending Blumer's theory of group position to a multiracial social context. Am Sociol Rev 61:951-972

Bosson JK, Vandello JA, Michniewicz KS, Lenes JG (2012) American men's and women's beliefs about gender discrimination: for men, it's not quite a zerosum game. Masc Soc Change 1(3):210-239. doi:10.4471/MCS.2012.14

Brinkley CA, Diamond PM, Magaletta PR, Heigel CP (2008) Cross-validation of Levenson's Psychopathy Scale in a sample of federal female inmates. Assessment 15(4):464-482. doi:10. $1177 / 1073191108319043$

De Dreu CK, Boles TL (1998) Share and share alike or winner take all?: The influence of social value orientation upon choice and recall of negotiation heuristics. Organ Behav Hum Decis Process 76(3):253-276

Esses VM, Dovidio JF, Jackson LM, Armstrong TL (2001) The immigration dilemma: the role of perceived group competition, ethnic prejudice, and national identity. J Soc Issues 57:389-412

Gries PH (2005) Social psychology and the identity-conflict debate: is a "China threat" inevitable? Eur J Int Relat 11:235-265

Hamilton EJ, Rathbun BC (2013) Scarce differences: toward a material and systemic foundation for offensive and defensive realism. Secur Stud 22(3):436-465

Hare RD (1991) The hare psychopathy checklist-revised (PCL-R). Multi-Health Syst, Toronto

Hare RD, Harpur TJ, Hemphill JD (1989) Scoring pamphlet for the Self-Report Psychopathy scale: SRPII. Unpublished manuscript, Simon Fraser University, Vancouver, British Columbia, Canada

Ho AK, Sidanius J, Pratto F, Levin S, Thomsen L, Kteily N, Sheehy-Skeffington J (2012) Social dominance orientation: revisiting the structure and function of a variable predicting social and political attitudes. Personal Soc Psychol Bull 38:583-606

John OP, Donahue EM, Kentle RL (1991) The big five inventory-versions 4a and 54. University of California, Berkeley, Institute of Personality and Social Research, Berkeley

Kalgaard R (2006) World's worst disease. Forbes Business Channel. http://www.forbes.com/global/2006/ 0109/035A.html

Kehn A, Ruthig JC (2013) Perceptions of gender discrimination across six decades: the moderating roles of gender and age. Sex Roles 69:289-296. doi:10.1007/s11199-013-0303-2

Lester WS, Salekin RT, Sellbom M (2012) The SRP-II as a rich source of data on the psychopathic personality. Psychol Assess 25(1):32-46. doi:10.1037/a0029449

Levenson MR, Kiehl KA, Fitzpatrick CM (1995) Assessing psychopathic attributes in a noninstitutionalized population. J Personal Soc Psychol 68:151-158

McCrae RR, John OP (1992) An introduction to the five-factor model and its applications. J Personal 60(2):175-215

Meegan DV (2010) Zero-sum bias: perceived competition despite unlimited resources. Front Psychol Cognit 1:1-7

Norton MI, Sommers SR (2011) Whites see racism as a zero-sum game that they are now losing. Perspect Psychol Sci 6:215-218. doi:10.1177/1745691611406922

Perry R, Sibley C, Duckitt J (2013) Dangerous and competitive worldviews: a meta-analysis of their associations with Social Dominance Orientation and Right-Wing Authoritarianism. J Res Personal 47:116-127

Ross L, Stillinger C (1991) Barriers to conflict resolution. Negoti J 7(4):389-404

Różycka-Tran J, Boski P, Wojciszke B (2015) Belief in a zero-sum game as a social axiom: a 37-nation study. J Cross Cult Psychol 46(4):525-548

Sellbom M, Lilienfeld SO, Fowler KA, McCrary KL (2017) The self-report assessment of psychopathy: challenges, pitfalls, and promises. In: Patrick CJ (ed) Handbook of psychopathy, 2nd edn. Guilford Press, New York (in press)

Shou Y, Sellbom M, Han J (2016) Evaluating the construct validity of the levenson self-report psychopathy scale in China. Assessment. doi:10.1177/1073191116637421

Smithson M, Shou Y (2016) Asymmetries in responses to attitude statements: the example of "zero-sum" beliefs. Front Psychol 7:984. doi:10.3389/fpsyg.2016.00984 
Smithson M, Sopeña A, Platow MJ (2015) When is group membership zero-sum? Effects of ethnicity, threat, and social identity on dual national identity. PLoS One 10(6):e0130539. doi:10.1371/journal. pone.0130539

Wiggins JS (ed) (1996) The five-factor model of personality: theoretical perspectives. Guilford Press, New York

Wilkins CL, Wellman JD, Babbitt LG, Toosi NR, Schad KD (2015) You can win but I can't lose: bias against high-status groups increases their zero-sum beliefs about discrimination. J Exp Soc Psychol 57:1-14

Wright R (2000) Nonzero: the logic of human destiny. Vintage Books, New York

Yee TW (2010) The VGAM package for categorical data analysis. J Stat Softw 32:1-34

Yu A (2014) Zero sum thinking and individual differences. Unpublished honours thesis, Canberra, Australia

Zimprich D, Perren S, Hornung R (2005) A two-level confirmatory factor analysis of a modified Rosenberg self-esteem scale. Educ Psychol Meas 65(3):465-481 